九州大学学術情報リポジトリ

Kyushu University Institutional Repository

\title{
Type-I Heterostructure and Improved Phase Stability of Formamidinium Lead Iodide Perovskite Grown on WS 2
}

Erkılıç, Ufuk

Interdisciplinary Graduate School of Engineering Sciences, Kyushu University

Ago, Hiroki

Interdisciplinary Graduate School of Engineering Sciences, Kyushu University

https://doi.org/10.5109/4068610

出版情報：Evergreen. 7 (3)，pp.323-328，2020-09. 九州大学グリーンテクノロジー研究教育センター バージョン：

権利関係 : 


\title{
Type-I Heterostructure and Improved Phase Stability of Formamidinium Lead Iodide Perovskite Grown on $\mathbf{W S}_{2}$
}

\author{
Ufuk Erk1liç ${ }^{1}$, Hiroki Ago ${ }^{1,2, *}$ \\ ${ }^{1}$ Interdisciplinary Graduate School of Engineering Sciences, Kyushu University, Japan \\ ${ }^{2}$ Global Innovation Center (GIC), Kyushu University, Japan \\ *Author to whom correspondence should be addressed: \\ E-mail: h-ago@gic.kyushu-u.ac.jp
}

(Received July 7, 2020; Revised September 7, 2020; accepted September 10, 2020).

\begin{abstract}
Heterostructures of two-dimensional materials provide an opportunity for the discovery of novel properties and improved material performances. In this work, we demonstrate the chemical vapor deposition growth of two-dimensional perovskite/ $\mathrm{WS}_{2}$ heterostructures. Owing to type-I band alignment, photoluminescence (PL) measurements indicated the photogenerated carrier transfer from the $\mathrm{WS}_{2}$ to the perovskite layer. In order to investigate the carrier transfer mechanism, power-dependent PL spectra were measured. The PL intensity of the two-dimensional perovskite grown on $\mathrm{WS}_{2}$ exhibited super-linear dependence to the excitation power. In addition, twodimensional perovskites grown on $\mathrm{WS}_{2}$ showed better air stability compared with the pristine perovskite. The improved stability is attributed to strain in the two-dimensional perovskite layer induced by the underlying $\mathrm{WS}_{2}$. The results presented here offer better understanding of the twodimensional perovskite based heterostructures and their potential use in practical applications, such as light emitting diodes and lasers.
\end{abstract}

Keywords: two-dimensional materials; hybrid halide perovskites; heterostructure, $\mathrm{WS}_{2}$

\section{Introduction}

Low-dimensional materials, such as nanoparticles, nanotubes, and nanosheets, offer great potential compared to their bulk counterparts ${ }^{1-4)}$. In particular, twodimensional (2D) materials have been extensively studied due to their unique and diverse properties ${ }^{5,6)}$. The most well-known 2D materials are graphene, transition metal dichalcogenides (TMDCs), and hexagonal boron nitride $(\mathrm{h}-\mathrm{BN})^{7}$. Recently, 2D hybrid halide perovskites have been reported as a new addition to the $2 \mathrm{D}$ materials family ${ }^{8)}$. 2D hybrid halide perovskites are a subclass of the hybrid halide perovskite family, which have a chemical formula $\mathrm{ABX}_{3}$, where $\mathrm{A}$ is an organic amine cation, $\mathrm{B}$ is a metal cation, and $\mathrm{X}$ is a halide anion ${ }^{9}$. Within a decade, these perovskites have shown promising applications in solar cells ${ }^{10)}$, photodetectors ${ }^{11)}$, and light emitting diodes $\left(\right.$ LEDs) ${ }^{12)}$. Compared to their bulk counterparts, 2D hybrid halide perovskites have several advantages, such as better stability, bandgap tuning by thickness engineering, and higher exciton binding energy ${ }^{8)}$. In addition, 2D perovskites exhibit novel properties due to strong quantum confinement effect ${ }^{13)}$.

Van der Waals heterostructures of 2D materials offer new phenomena in next generation device applications, such as interlayer excitons, valleytronics, and moiré potentials ${ }^{14-16)}$. Recently, heterostructures of 2D perovskite with other 2D materials have been reported. These heterostructures exhibited improved performances in device applications and novel properties. Very recently, we demonstrated a perovskite/TMDC heterostructure with type-II band alignment ${ }^{17}$. In type-II heterostructures, electrons and holes are separated into different layers due to the staggered alignment of valance and conduction bands of each layer. Type-II heterostructures of 2D materials have been widely studied for charge separation, which is an important phenomenon for solar cells ${ }^{18)}$. On the other hand, in type-I band alignment electrons and holes are confined in the small bandgap material, which are transferred from the large bandgap material. These confined charge carriers (electrons and holes) in heterostructures with type-I band alignment offer a great potential in optoelectronic applications, such as LEDs ${ }^{19)}$. However, type-I heterostructures based on 2D materials have been scarce.

In this work, we report a 2D perovskite/tungsten disulfide $\left(\mathrm{WS}_{2}\right)$ heterostructure with type-I band alignment. We employed a small bandgap 2D perovskite, namely, formamidinium lead iodide $\left(\mathrm{FAPbI}_{3}\right)$. $\mathrm{FAPbI}_{3} / \mathrm{WS}_{2}$ heterostructures were fabricated by chemical vapor deposition (CVD) methods. The photoluminescence (PL) measurement of the $\mathrm{FAPbI}_{3} / \mathrm{WS}_{2}$ heterostructure revealed a PL enhancement in the $\mathrm{FAPbI}_{3}$ emission, while $\mathrm{WS}_{2}$ peak showed PL 

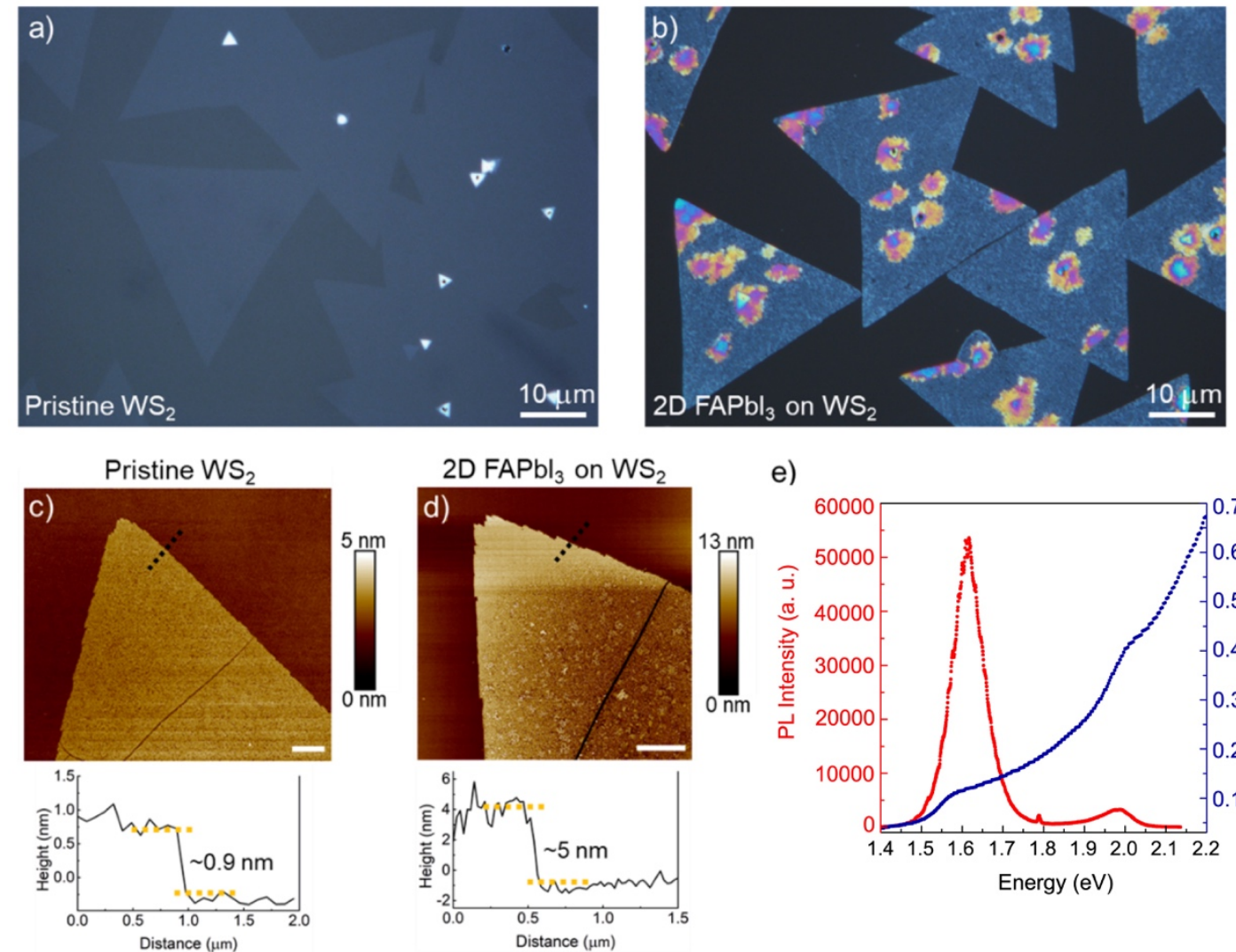

2D $\mathrm{FAPbl}_{3}$ on $\mathrm{WS}_{2}$
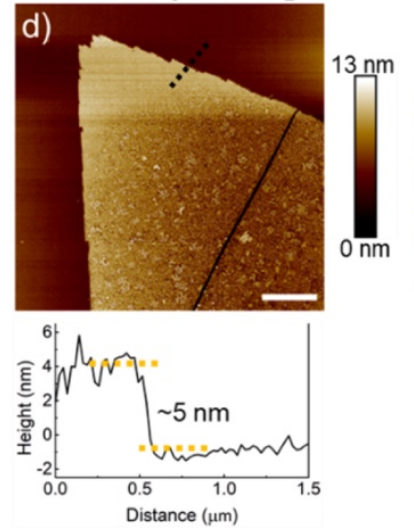

e)

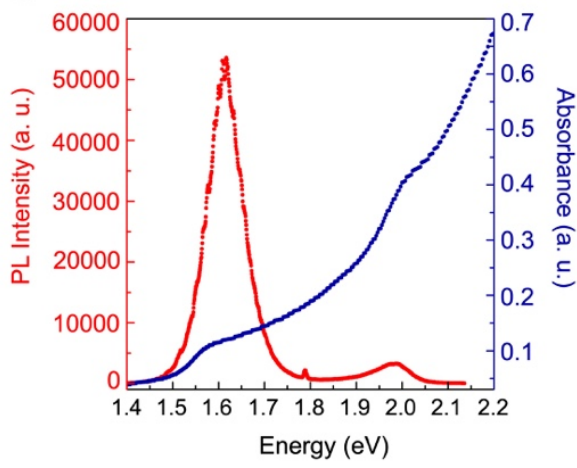

Fig. 1: Optical images of (a) as-grown $\mathrm{WS}_{2}$ grain with a triangular shape and same grain after the deposition of a 2D FAPbI3

(b). AFM images of pristine $\mathrm{WS}_{2}$ (c), and $\mathrm{FAPbI}_{3} / \mathrm{WS}_{2}$ heterostructure (d). Scale bars are $2 \mu \mathrm{m}$. Lower panels of AFM images are the height profiles measured along the black dashed lines shown in the corresponding AFM images. (d) PL (red data) and absorption (blue data) spectra of the $\mathrm{FAPbI}_{3} / \mathrm{WS}_{2}$ heterostructure.

quenching. This is attributed to the transfer of photogenerated carriers from the $\mathrm{WS}_{2}$ layer to $\mathrm{FAPbI}_{3}$ because of type-I band alignment. Power dependent PL measurement also supported the transfer of both electrons and holes from $\mathrm{WS}_{2}$ to $\mathrm{FAPbI}_{3}$. In addition, the $\mathrm{FAPbI}_{3}$ grown on $\mathrm{WS}_{2}$ showed higher stability over long time compared to $\mathrm{FAPbI}_{3}$ only sample. We discuss the origin of this improved stability in detail.

\section{Experimental}

\subsection{CVD growth of $\mathrm{FAPbI}_{3} / \mathrm{WS}_{2}$}

First, monolayer $\mathrm{WS}_{2}$ was synthesized by CVD on cplane sapphire, as reported previously from our group ${ }^{20)}$. $\mathrm{WO}_{3}$ and sulfur powders were placed in a three-zone furnace. The $\mathrm{WO}_{3}$, sulfur, and substrates were heated at $1065^{\circ} \mathrm{C}, 165^{\circ} \mathrm{C}$, and $925^{\circ} \mathrm{C}$, respectively. This as-grown $\mathrm{WS}_{2} /$ sapphire substrate was subjected to the following two-step process ${ }^{17}$ ) to synthesize $\mathrm{FAPbI}_{3}$ perovskite layer on the $\mathrm{WS}_{2}$ surface. First step was the vapor-deposition of $\mathrm{PbI}_{2}$ on $\mathrm{WS}_{2}$. For this, $\mathrm{PbI}_{2}$ powder was placed at the center of a CVD furnace, and as-grown $\mathrm{WS}_{2} /$ sapphire substrate was put at the downstream. While flowing 50 $\mathrm{ccm}$ of Ar gas, the furnace temperature was gradually increased to $360{ }^{\circ} \mathrm{C}$ and kept at this temperature for 15 min to deposit $\mathrm{PbI}_{2}$ on $\mathrm{WS}_{2}$. $\mathrm{PbI}_{2}$ was then converted to perovskite $\left(\mathrm{FAPbI}_{3}\right)$ by intercalating formamidinium iodide at $150{ }^{\circ} \mathrm{C}$ for 20 min under a constant Ar flow.

\subsection{Characterizations}

Optical images were taken by an optical microscope (Nikon Eclipse ME600). Atomic force microscope (AFM) images were obtained using a Nanoscope V (Bruker). PL spectra were collected with a Raman spectroscope (Tokyo Instruments Nanofinder 30) using a 532 nm laser. UV-vis absorption spectra were measured by using a UV-3600 (Shimadzu) spectrophotometer.

\section{Results and discussion}

Figure $1 \mathrm{a}$ and $\mathrm{b}$ shows the optical images of monolayer $\mathrm{WS}_{2}$ before and after the deposition of a $2 \mathrm{D} \mathrm{FAPbI}_{3}$ layer, respectively. As-grown $\mathrm{WS}_{2}$ grains have triangular shapes, with some of the grains merging with each other. White contrast seen at the center of some grains is multilayer $\mathrm{WS}_{2}$ which acted as nucleation cite. As can be seen in Fig. $1 \mathrm{~b}, \mathrm{FAPbI}_{3}$ perovskite layer selectively grew on the $\mathrm{WS}_{2}$. Previously, we reported this unique selective growth behavior for $\mathrm{MAPbI}_{3}$ perovskite/WS $\mathrm{WS}_{2}$ heterostructures. ${ }^{17)}$ Our analyses revealed that the higher surface energy of 

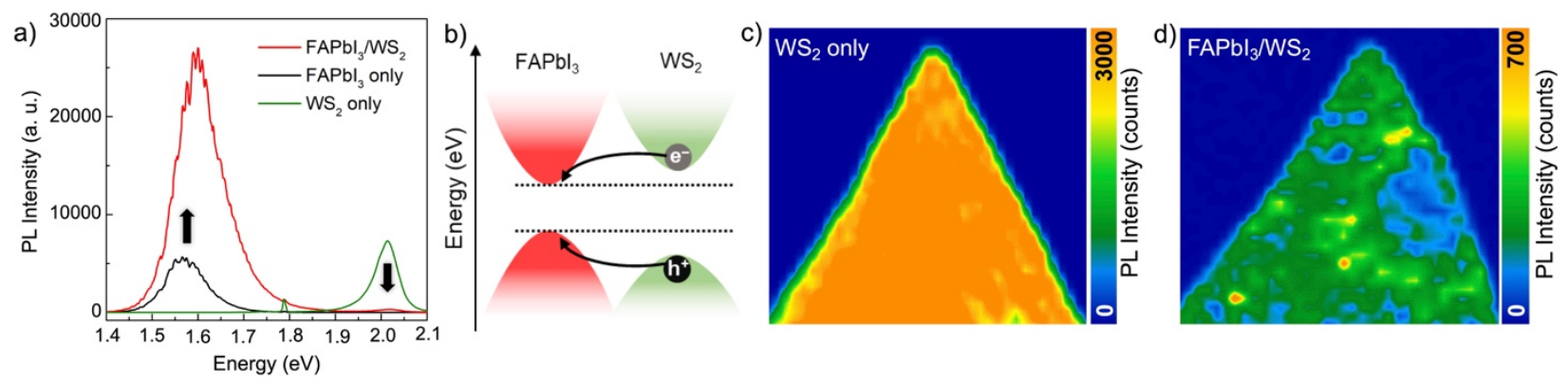

Fig. 2: (a) PL spectra of the heterostructure (red line), $\mathrm{FAPbI}_{3}$ only (black line), and $\mathrm{WS}_{2}$ only samples (green line). (b) Band diagrams of $\mathrm{FAPbI}_{3}$ and $\mathrm{WS}_{2}$, showing the type-I band alignment. PL intensity mappings for $\mathrm{WS}_{2}$ peak (2 eV) for WS $\mathrm{W}_{2}$ only (c), and $\mathrm{FAPbI}_{3} / \mathrm{WS}_{2}(\mathrm{~d})$.

$\mathrm{WS}_{2}$ compared to c-plane sapphire assists the growth of 2D perovskite layer ${ }^{17)}$. As the selective growth takes place during the deposition of the $\mathrm{PbI}_{2}$ layer, we observe a similar growth behavior in $\mathrm{FAPbI}_{3}$. Here, the higher surface energy of $\mathrm{WS}_{2}$ compensates the energy barrier required for the nucleation of $\mathrm{PbI}_{2}$. In Fig. 1b, the red and yellow colored areas correspond to thick perovskite layers that grew around the nucleation centers of $\mathrm{WS}_{2}$ grains. The AFM images of the $\mathrm{WS}_{2}$ and $\mathrm{FAPbI}_{3} / \mathrm{WS}_{2}$ heterostructure are shown in Fig. 1c and d, respectively. Pristine $\mathrm{WS}_{2}$ has a thickness around $0.9 \mathrm{~nm}$, which agrees well with the reported value ${ }^{21)}$. In the case of the heterostructure (Fig. 1d), total thickness increased to $\sim 5$ $\mathrm{nm}$, indicating that $\mathrm{FAPbI}_{3}$ thickness is around $4 \mathrm{~nm}$. These results confirm the deposition of a 2D thin layer of $\mathrm{FAPbI}_{3}$ on $\mathrm{WS}_{2}$. To further verify the formation of the heterostructure, PL and absorption spectra were also measured (see Fig. 1e). PL spectrum of the heterostructure (red data in Fig. 1e) consists of two distinct PL peaks. The PL peak at $1.61 \mathrm{eV}$ is originated in the $2 \mathrm{D} \mathrm{FAPbI}_{3}$, while the peak at $2 \mathrm{eV}$ is the emission from $\mathrm{WS}_{2}$ layer ${ }^{20)}$. For bulk $\mathrm{FAPbI}_{3}$ perovskites optical bandgap is around 1.55 $\mathrm{eV}^{22)}$. However, in our case the bandgap increased to 1.61 $\mathrm{eV}$ as can be seen in the blue shifted PL emission. This blue shift can be explained by the quantum confinement effect in 2D $\mathrm{FAPbI}_{3}$ due to the reduced thickness ${ }^{17,23)}$. Absorption spectrum of the heterostructure (blue data in Fig 1e) agrees well with the PL spectrum. A sharp absorption edge at $\sim 1.58 \mathrm{eV}$ arises from $\mathrm{FAPbI}_{3}$, while additional absorption edge at $\sim 2 \mathrm{eV}$ is from the $\mathrm{WS}_{2}$ layer.

To further elaborate the optical properties, PL spectra of isolated $\mathrm{FAPbI}_{3}$ and $\mathrm{WS}_{2}$ were compared with that of the heterostructure in Fig. 2a. As expected, the pristine $\mathrm{FAPbI}_{3}$ and the pristine $\mathrm{WS}_{2}$ showed PL emission at 1.58 $\mathrm{eV}$ and $2 \mathrm{eV}$, respectively. Interestingly, in the case of the heterostructure, the PL intensity of $\mathrm{FAPbI}_{3}$ considerably increased. In contrast, the PL from $\mathrm{WS}_{2}$ was strongly quenched. According to the band structure calculations, the heterostructure of $\mathrm{FAPbI}_{3}$ and $\mathrm{WS}_{2}$ possesses type-I band alignment ${ }^{17,24-26)}$. The type-I band alignment is displayed in Fig. 2b. In this type-I alignment, photogenerated charge carriers (both electrons and holes) transfer from the large bandgap material $\left(\mathrm{WS}_{2}\right)$ to the small bandgap material $\left(\mathrm{FAPbI}_{3}\right)$. Therefore, the increased PL intensity of $\mathrm{FAPbI}_{3}$ in the heterostructure can be explained by energy transfer from the $\mathrm{WS}_{2}$ layer to the $\mathrm{FAPbI}_{3}$ layer upon photoexcitation ${ }^{19)}$. PL peak intensity mappings for $\mathrm{WS}_{2}$ peak measured before and after $\mathrm{FAPbI}_{3}$ deposition are shown in Fig. 2c and d, respectively. It is clear that after the $\mathrm{FAPbI}_{3}$ deposition, the PL intensity of the $\mathrm{WS}_{2}$ decreased on the whole grain. We note that deposition of another material can cause a decrease in the PL intensity by preventing the laser penetration. However, considering the optical absorption coefficient of $\mathrm{FAPbI}_{3}$ (up to $10^{5} \mathrm{~cm}^{-1}$ ) 27 ), penetration depth for a $532 \mathrm{~nm}$ laser is around $100 \mathrm{~nm}$. Therefore, we excluded the screening effect by $\mathrm{FAPbI}_{3}$ on the $\mathrm{PL}$ quenching of $\mathrm{WS}_{2}$ peak.

In order to understand the increased PL intensity of $\mathrm{FAPbI}_{3}$ in the heterostructure, we measured excitation power dependence of the PL spectrum. In Fig. 3,

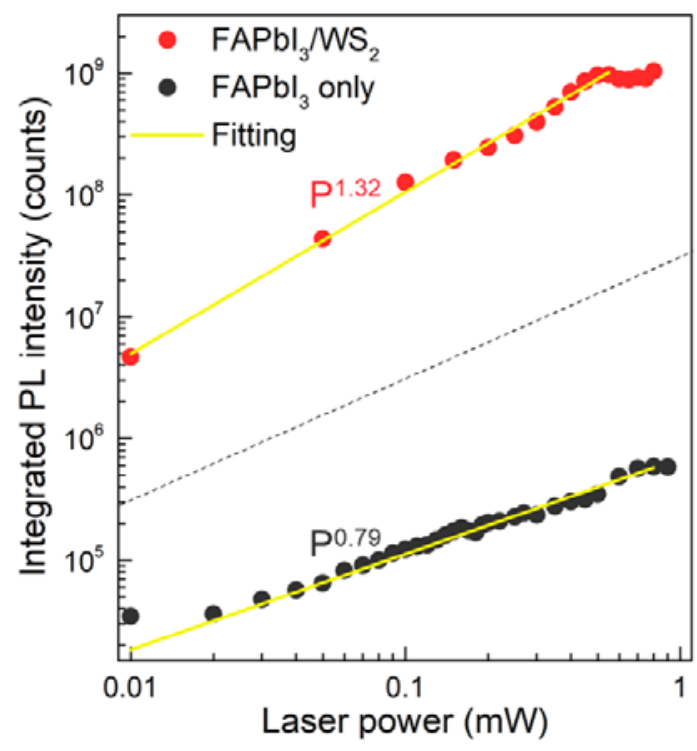

Fig. 3: Log-log plot of laser power dependence of integrated $\mathrm{PL}$ intensity for $\mathrm{FAPbI}_{3}$ peak in the heterostructure (red dots) and in the FAPbI 3 only sample. PL peak intensities are integrated over the range of 1.45$1.75 \mathrm{eV}$ for the $\mathrm{FAPbI}_{3}$ peak. Yellow lines correspond to the power-law fittings. Black dotted line is the linear power dependence, which is added as a guide for comparing super-linear and sublinear power dependence. 

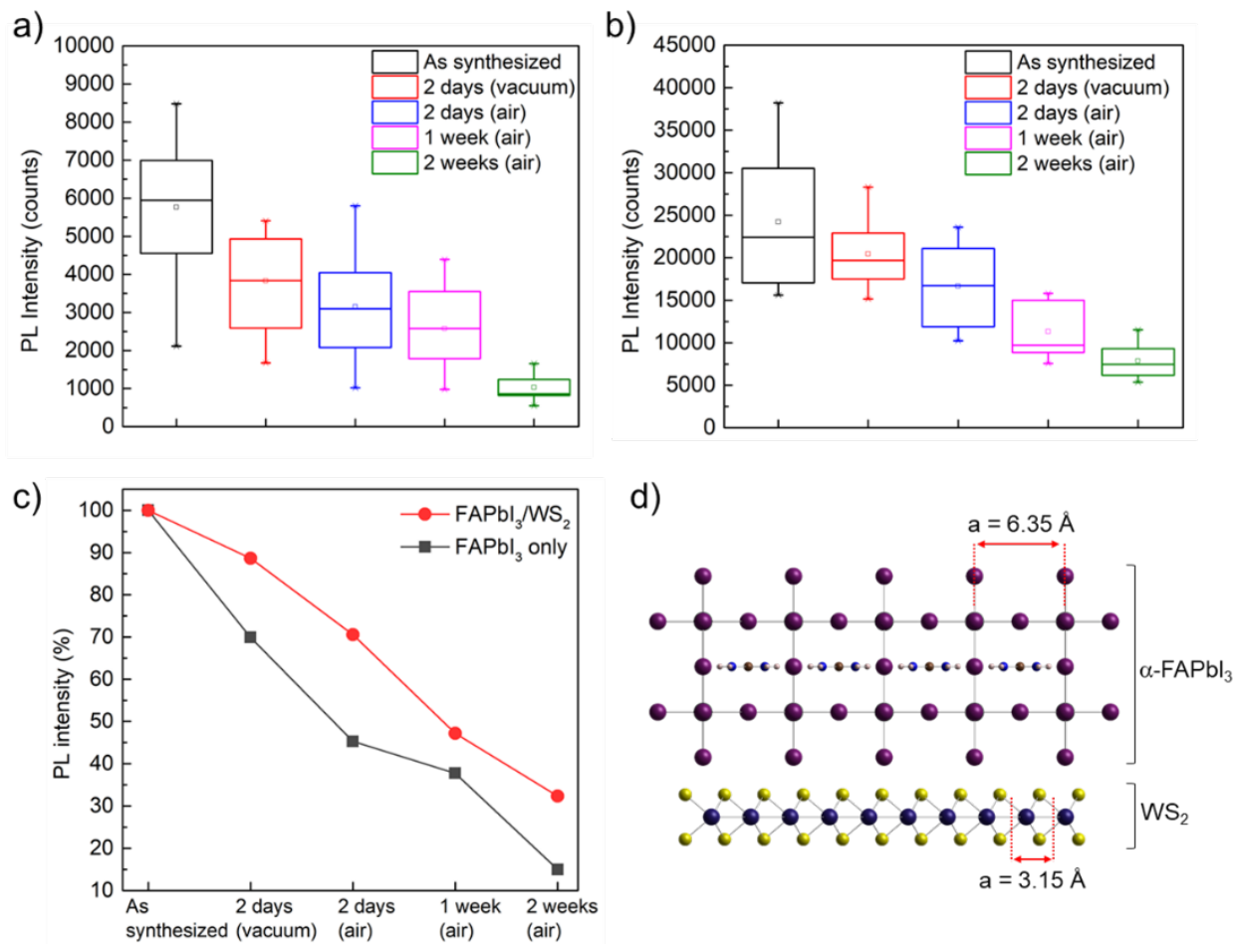

Fig. 4: PL intensity measurements for $\mathrm{FAPbI}_{3}$ only (a), and $\mathrm{FAPbI}_{3} / \mathrm{WS}_{2}$ for up to 2 weeks in air. (c) Comparison of intensity decrease in $\mathrm{FAPbI}_{3} \mathrm{PL}$ emission for $\mathrm{FAPbI}_{3}$ only (black line) and $\mathrm{FAPbI}_{3} / \mathrm{WS}_{2}$ (red line). (d) An atomic model of $\mathrm{FAPbI}_{3} / \mathrm{WS}_{2}$ heterostructure.

integrated $\mathrm{PL}$ intensity of the $\mathrm{FAPbI}_{3}$ peak is plotted as a function of the laser power. Fig. 3 displays the power dependence of the PL intensity measured for $\mathrm{FAPbI}_{3}$ only peak (black dots) and $\mathrm{FAPbI}_{3}$ peak in the heterostructures (red dots). The data can be fitted by a power-law function ( $I \propto P^{k}$ ), where $I$ is the PL intensity, $P$ is the excitation power, and $k$ is the power factor ${ }^{28)}$. Depending on the charge carrier recombination mechanism, different $k$ values can be observed. $k<1$ is indicative of free-tobound transitions or Auger process, while $1<k<2$ is for excitonic or biexcitonic transitions ${ }^{29)}$. Power factors were calculated based on power-law function fittings shown by yellow lines in Fig. 3. For the perovskite only peak, $k=$ 0.79 refers to the recombination of free carrier or Auger recombination ${ }^{30)}$. For the $\mathrm{FAPbI}_{3}$ peak in the heterostructure, a super-linear power dependence with $k=$ 1.32 was observed. This super-linear dependence can be related to recombination of mixed free carrier and excitonic states. This sublinear $(k=0.79)$ to super-linear $(k=1.32)$ transition can be attributed to the disassociation of excitonic species at the $\mathrm{FAPbI}_{3} / \mathrm{WS}_{2}$ interface as a result of carrier transfer in type-I band alignment ${ }^{30,31)}$. However, the charge carrier dynamics of the $\mathrm{FAPbI}_{3} / \mathrm{WS}_{2}$ heterostructure need to be further investigated.

Finally, long-term PL stability of the heterostructure was also investigated. In Fig. 4a and b, PL spectra of $\mathrm{FAPbI}_{3}$ only and $\mathrm{FAPbI}_{3}$ grown on $\mathrm{WS}_{2}$ were measured for up to two weeks. In both cases, the PL intensity decreased with increasing the time. It is known that $\mathrm{FAPbI}_{3}$ undergoes a phase transformation at room temperature ${ }^{20)}$. $\alpha$-phase of $\mathrm{FAPbI}_{3}$ is the desired perovskite structure. However, $\alpha-\mathrm{FAPbI}_{3}$ is unstable at room temperature and it gradually converts to nonperovskite $\delta$-phase structure ${ }^{22,32)}$. The $\delta$-phase of $\mathrm{FAPbI}_{3}$ is undesirable as it does not show PL emission. Therefore, the decrease in the PL intensity is a sign of phase transformation of $\mathrm{FAPbI}_{3}$ into non-perovskite $\delta$-phase. In Fig. 4c, decrease in the PL intensity of $\mathrm{FAPbI}_{3}$ only (black line) and $\mathrm{FAPbI}_{3}$ grown on $\mathrm{WS}_{2}$ (red line) is compared. In the case of $\mathrm{FAPbI}_{3}$ only sample, PL intensity decreased almost $85 \%$ with respect to the initial intensity. For $\mathrm{FAPbI}_{3}$ grown on $\mathrm{WS}_{2}$, PL intensity only decreased to $\sim 67 \%$. The better stability of the latter can be attributed to the slower phase transformation of $\alpha-\mathrm{FAPbI}_{3}$ into $\delta$ $\mathrm{FAPbI}_{3}$. It is likely that $\mathrm{WS}_{2}$ layer suppresses the structural transformation of $\mathrm{FAPbI}_{3}$. Very recently, Chen et al., reported the phase stabilization of $\mathrm{FAPbI}_{3}$ through strain engineering ${ }^{33)}$. They claimed that strain is induced by heteroepitaxial growth of $\mathrm{FAPbI}_{3}$ on lattice mismatched substrates, which stabilizes the $\alpha$-FAPbI structure. Previously we also reported the epitaxial growth of $2 \mathrm{D}$ perovskite on $\mathrm{WS}_{2}{ }^{17)}$. Therefore, most likely $\mathrm{WS}_{2}$ may induce a strain on $\mathrm{FAPbI}_{3}$, which stabilizes the $\alpha$ phase by preventing the phase transformation. In Fig. 4d, an atomic model of $\mathrm{FAPbI}_{3} / \mathrm{WS}_{2}$ heterostructure is shown. The $\alpha-\mathrm{FAPbI}_{3}$ has a larger lattice constant $(6.35 \AA)^{33)}$ compared to $\mathrm{WS}_{2}(3.15 \AA)^{34)}$. Therefore, this large lattice mismatch is expected to cause a compressive strain on the $\alpha$-FAPbI 3 lattice. As conversion to $\delta$-FAPbI 3 is triggered by the internal tensile strain, the compressive strain caused by $\mathrm{WS}_{2}$ cancels out the internal tensile strain in $\alpha$-FAPbI 3 . 


\section{Conclusions}

We present the CVD growth of a 2D heterostructure of $\mathrm{FAPbI}_{3}$ and $\mathrm{WS}_{2}$. Type-I band alignment in the heterostructure enhanced the PL emission from the $\mathrm{FAPbI}_{3}$ layer as a result of photogenerated energy transfer from the monolayer $\mathrm{WS}_{2}$ to the $\mathrm{FAPbI}_{3}$ layer. The power dependent PL measurements for $\mathrm{FAPbI}_{3}$ in the heterostructure showed a super-linear dependence, which indicates the excitonic recombination at the $\mathrm{FAPbI}_{3} / \mathrm{WS}_{2}$ interface. These results hold a promise for realizing typeI device structure based on 2D perovskites. Type-I heterostructures are promising in LED and laser applications due to the enhanced light-matter interactions because of the confined charge carriers. Finally, the heterostructure showed an improved phase stability of $\alpha$ $\mathrm{FAPbI}_{3}$, likely due to $\mathrm{WS}_{2}$-induced strain. As the longterm stability of perovskites is a major concern, we believe that our result would also contribute to the stabilization studies of perovskite structures.

\section{Acknowledgements}

This work was supported by the JSPS KAKENHI grant numbers JP19K22113 and JP18H03864, JST CREST grant numbers JPMJCR18I1, and JSPS A3 Foresight Program. U.E. acknowledges the Intellectual Exchange and Innovation (IEI) program supported by MEXT.

\section{References}

1) A.M.E. Khalil, O. Eljamal, R. Eljamal, Y. Sugihara, and N. Matsunaga, "Treatment and regeneration of nano-scale zero-valent iron spent in water remediation," Evergreen, 4 (1) 21-28 (2017). doi:10.5109/1808449.

2) M. Egiza, H. Naragino, A. Tominaga, K. Murasawa, H. Gonda, M. Sakurai, and T. Yoshitake, "Si and Cr doping effects on growth and mechanical properties of ultrananocrystalline diamond/amorphous carbon composite films deposited on cemented carbide substrates by coaxial arc plasma deposition," Evergreen, 3 (1) 32-36 (2016). doi:10.5109/1657738.

3) R. Ruliandini, and T. Tokumasu, "Assessing hBN nanoparticles stability in trimethylolpropane triester based biolubricants using molecular dynamic simulation,” Evergreen, 7 (2) 234-239 (2020).

4) P.P.D.K. Wulan, J.A. Ningtyas, and M. Hasanah, "The effect of nickel coating on stainless steel 316 on growth of carbon nanotube from polypropylene waste," Evergreen, 6 (1) 98-102 (2019). doi:10.5109/2328411.

5) P. Solís-Fernández, M. Bissett, and H. Ago, "Synthesis, structure and applications of graphenebased 2D heterostructures,” Chem. Soc. Rev., 46 (15) 4572-4613 (2017). doi:10.1039/c7cs00160f.

6) M.S. Rahman, T. Nakagawa, and S. Mizuno,
"Germanene: experimental study for graphene like two dimensional germanium,” Evergreen, 1 (2) 2529 (2014). doi:10.5109/1495160.

7) G.R. Bhimanapati, Z. Lin, V. Meunier, Y. Jung, J. Cha, S. Das, D. Xiao, Y. Son, M.S. Strano, V.R. Cooper, L. Liang, S.G. Louie, E. Ringe, W. Zhou, S.S. Kim, R.R. Naik, B.G. Sumpter, H. Terrones, F. Xia, Y. Wang, J. Zhu, D. Akinwande, N. Alem, J.A. Schuller, R.E. Schaak, M. Terrones, and J.A. Robinson, "Recent advances in two-dimensional materials beyond graphene,” ACS Nano, 9 (12) 11509-11539 (2015). doi:10.1021/acsnano.5b05556.

8) C. Huo, B. Cai, Z. Yuan, B. Ma, and H. Zeng, "Twodimensional metal halide perovskites: theory, synthesis, and optoelectronics,” Small Methods, 1 (3) 1600018 (2017). doi:10.1002/smtd.201600018.

9) Y. Zhang, J. Liu, Z. Wang, Y. Xue, Q. Ou, L. Polavarapu, J. Zheng, X. Qi, and Q. Bao, “Synthesis, properties, and optical applications of lowdimensional perovskites," Chem. Commun., 52 (94) 13637-13655 (2016). doi:10.1039/c6cc06425f.

10) G. Grancini, C. Roldán-Carmona, I. Zimmermann, E. Mosconi, X. Lee, D. Martineau, S. Narbey, F. Oswald, F. De Angelis, M. Graetzel, and M.K. Nazeeruddin, “One-year stable perovskite solar cells by 2D/3D interface engineering,” Nat. Commun., 8 (1) 15684 (2017). doi:10.1038/ncomms15684.

11) H. Wang, and D.H. Kim, "Perovskite-based photodetectors: materials and devices," Chem. Soc. Rev., $46 \quad$ (17) 5204-5236 (2017). doi:10.1039/c6cs00896h.

12) X. Yang, X. Zhang, J. Deng, Z. Chu, Q. Jiang, J. Meng, P. Wang, L. Zhang, Z. Yin, and J. You, "Efficient green light-emitting diodes based on quasi-twodimensional composition and phase engineered perovskite with surface passivation," Nat. Commun., 9 (1) 570 (2018). doi:10.1038/s41467-018-02978-7.

13) U. Erkılıç, H. G. Ji, E. Nishibori, H. Ago, “One-step vapour phase growth of two-dimensional formamidinium-based perovskite and its hot carrier dynamics,” Phys. Chem. Chem. Phys., (2020). doi: 10.1039/D0CP02652B

14) P. Rivera, J.R. Schaibley, A.M. Jones, J.S. Ross, S. Wu, G. Aivazian, P. Klement, K. Seyler, G. Clark, N.J. Ghimire, J. Yan, D.G. Mandrus, W. Yao, and X. Xu, "Observation of long-lived interlayer excitons in monolayer $\mathrm{MoSe}_{2}-\mathrm{WSe}_{2}$ heterostructures," Nat. Commun., $6 \quad$ (1) $6242 \quad$ (2015). doi:10.1038/ncomms7242.

15) P. Nagler, M. V Ballottin, A.A. Mitioglu, F. Mooshammer, N. Paradiso, C. Strunk, R. Huber, A. Chernikov, P.C.M. Christianen, C. Schüller, and T. Korn, "Giant magnetic splitting inducing near-unity valley polarization in van der Waals heterostructures," Nat. Commun., $8 \quad$ (1) $1551 \quad$ (2017). doi:10.1038/s41467-017-01748-1.

16) C. Zhang, C.-P. Chuu, X. Ren, M.-Y. Li, L.-J. Li, C. Jin, M.-Y. Chou, and C.-K. Shih, "Interlayer 
couplings, moiré patterns, and 2D electronic superlattices in $\mathrm{MoS}_{2} / \mathrm{WSe}_{2}$ hetero-bilayers," Sci.

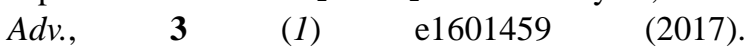
doi:10.1126/sciadv.1601459.

17) U. Erkılıç, P. Solís-Fernández, H.G. Ji, K. Shinokita, Y.-C. Lin, M. Maruyama, K. Suenaga, S. Okada, K. Matsuda, and H. Ago, "Vapor phase selective growth of two-dimensional perovskite/ $\mathrm{WS}_{2}$ heterostructures for optoelectronic applications," ACS Appl. Mater. Interfaces, 11 (43) 40503-40511 (2019). doi:10.1021/acsami.9b13904.

18) X. Hong, J. Kim, S.F. Shi, Y. Zhang, C. Jin, Y. Sun, S. Tongay, J. Wu, Y. Zhang, and F. Wang, "Ultrafast charge transfer in atomically thin $\mathrm{MoS}_{2} / \mathrm{WS}_{2}$ heterostructures,” Nat. Nanotechnol., 9 (9) 682-686 (2014). doi:10.1038/nnano.2014.167.

19) M.Z. Bellus, M. Li, S.D. Lane, F. Ceballos, Q. Cui, X.C. Zeng, and H. Zhao, "Type-I van der Waals heterostructure formed by $\mathrm{MoS}_{2}$ and $\mathrm{ReS}_{2}$ monolayers," Nanoscale Horizons, 2 (1) 31-36 (2017). doi:10.1039/C6NH00144K.

20) H.G. Ji, M. Maruyama, A.S. Aji, S. Okada, K. Matsuda, and H. Ago, "Van der Waals interactioninduced photoluminescence weakening and multilayer growth in epitaxially aligned $\mathrm{WS}_{2}$," Phys. Chem. Chem. Phys., 20 (47) 29790-29797 (2018). doi:10.1039/c8cp04418j.

21) K.N. Kang, K. Godin, and E.-H. Yang, "The growth scale and kinetics of $\mathrm{WS}_{2}$ monolayers under varying $\mathrm{H}_{2}$ concentration,” Sci. Rep., 5 (1) 13205 (2015). doi:10.1038/srep13205.

22) F. Ma, J. Li, W. Li, N. Lin, L. Wang, and J. Qiao, "Stable $\alpha / \delta$ phase junction of formamidinium lead iodide perovskites for enhanced near-infrared emission," Chem. Sci., 8 (1) 800-805 (2017). doi:10.1039/C6SC03542F.

23) J. Liu, Y. Xue, Z. Wang, Z.Q. Xu, C. Zheng, B. Weber, J. Song, Y. Wang, Y. Lu, Y. Zhang, and Q. Bao, “Twodimensional $\mathrm{CH}_{3} \mathrm{NH}_{3} \mathrm{PbI}_{3}$ perovskite: synthesis and optoelectronic application,” ACS Nano, 10 (3) 35363542 (2016). doi:10.1021/acsnano.5b07791.

24) A.R.B.M. Yusoff, and M.K. Nazeeruddin, "Organohalide lead perovskites for photovoltaic applications,” J. Phys. Chem. Lett., 7 (5) 851-866 (2016). doi:10.1021/acs.jpclett.5b02893.

25) T. Zhang, J. Wu, P. Zhang, W. Ahmad, Y. Wang, M. Alqahtani, H. Chen, C. Gao, Z.D. Chen, Z. Wang, and S. Li, "High speed and stable solution-processed triple cation perovskite photodetectors," Adv. Opt. Mater., $6 \quad$ (13) $1701341 \quad$ (2018). doi:10.1002/adom.201701341.

26) C. Zhang, C. Gong, Y. Nie, K.-A. Min, C. Liang, Y.J. Oh, H. Zhang, W. Wang, S. Hong, L. Colombo, R.M. Wallace, and K. Cho, "Systematic study of electronic structure and band alignment of monolayer transition metal dichalcogenides in van der Waals heterostructures,” 2D Mater., 4 (1) 015026 (2016). doi:10.1088/2053-1583/4/1/015026.

27) M. Kato, T. Fujiseki, T. Miyadera, T. Sugita, S. Fujimoto, M. Tamakoshi, M. Chikamatsu, and H. Fujiwara, "Universal rules for visible-light absorption in hybrid perovskite materials,” J. Appl. Phys., 121 (11) 115501 (2017). doi:10.1063/1.4978071.

28) H. Shibata, M. Sakai, A. Yamada, K. Matsubara, K. Sakurai, H. Tampo, S. Ishizuka, K.K. Kim, and S. Niki, "Excitation-power dependence of free exciton photoluminescence of semiconductors," Jpn. J. Appl. Phys., Part 1, 44 (8) 6113-6114 (2005). doi:10.1143/JJAP.44.6113.

29) S. Draguta, J.A. Christians, Y. V. Morozov, A. Mucunzi, J.S. Manser, P. V. Kamat, J.M. Luther, and M. Kuno, “A quantitative and spatially resolved analysis of the performance-bottleneck in high efficiency, planar hybrid perovskite solar cells," Energy Environ. Sci., 11 (4) 960-969 (2018). doi:10.1039/C7EE03654J.

30) A. Yang, J.-C. Blancon, W. Jiang, H. Zhang, J. Wong, E. Yan, Y.-R. Lin, J. Crochet, M.G. Kanatzidis, D. Jariwala, T. Low, A.D. Mohite, and H.A. Atwater, "Giant enhancement of photoluminescence emission in $\mathrm{WS}_{2}$-two-dimensional perovskite heterostructures," Nano Lett, $19 \quad$ (8) 4852-4860 (2019). doi:10.1021/acs.nanolett.8b05105.

31) G.E. Cirlin, V.G. Talalaev, N.D. Zakharov, V.A. Egorov, and P. Werner, "Room temperature superlinear power dependence of photoluminescence from defect-free $\mathrm{Si} / \mathrm{Ge}$ quantum dot multilayer structures,” Phys. Status Solidi Basic Res., 232 (1) R1-R3 (2002). doi:10.1002/15213951(200207)232:1<R1::AIDPSSB99991>3.0.CO;2-Z.

32) A. Binek, F.C. Hanusch, P. Docampo, and T. Bein, "Stabilization of the trigonal high-temperature phase of formamidinium lead iodide,” J. Phys. Chem. Lett., 6 (7) 1249-1253 (2015). doi:10.1021/acs.jpclett.5b00380.

33) Y. Chen, Y. Lei, Y. Li, Y. Yu, J. Cai, M.-H. Chiu, R. Rao, Y. Gu, C. Wang, W. Choi, H. Hu, C. Wang, Y. Li, J. Song, J. Zhang, B. Qi, M. Lin, Z. Zhang, A.E. Islam, B. Maruyama, S. Dayeh, L.-J. Li, K. Yang, Y.-H. Lo, and $\mathrm{S}$. $\mathrm{Xu}$, "Strain engineering and epitaxial stabilization of halide perovskites," Nature, 577 (7789) 209-215 (2020). doi:10.1038/s41586-0191868-X.

34) A. Berkdemir, H.R. Gutiérrez, A.R. Botello-Méndez, N. Perea-López, A.L. Elías, C.I. Chia, B. Wang, V.H. Crespi, F. López-Urías, J.C. Charlier, H. Terrones, and M. Terrones, "Identification of individual and few layers of $\mathrm{WS}_{2}$ using raman spectroscopy," Sci. Rep., 3 (June) 1755 (2013). doi:10.1038/srep01755. 